\title{
Emulsion-Oriented Assembly for Janus Double- Spherical Mesoporous Nanoparticles as Biological Logic Gates
}

\section{Tiancong Zhao}

Fudan University

\section{Liang Chen}

Shanghai University https://orcid.org/0000-0002-1437-4308

Minchao Liu

Fudan University

Runfeng Lin

Fudan University

\section{Weiluo Cai}

Shanghai Medical College, Fudan University

\section{Chinte Hung}

Fudan University

\section{Shangfeng Wang}

Fudan University

\section{Linlin Duan}

Fudan University

\section{Fan Zhang}

Fudan University https://orcid.org/0000-0001-7886-6144

\section{Ahmed Elzatahry}

Qatar University https://orcid.org/0000-0002-7291-5723

\section{Xiaomin Li}

Fudan University https://orcid.org/0000-0001-6056-6928

Dongyuan Zhao ( $\nabla$ dyzhao@fudan.edu.cn )

Fudan University https://orcid.org/0000-0001-8440-6902

\section{Article}

Keywords: biological logic systems, asymmetric nanomaterials, logic gates, mesoporous nanoparticles

Posted Date: October 25th, 2021

DOl: https://doi.org/10.21203/rs.3.rs-960508/v1 
License: (c) (i) This work is licensed under a Creative Commons Attribution 4.0 International License. Read Full License

Version of Record: A version of this preprint was published at Nature Chemistry on April 13th, 2023. See the published version at https://doi.org/10.1038/s41557-023-01183-4. 


\section{Abstract}

Mesoporous Janus nanoparticles, due to the independent mesoporous compartments, tunable spatial isolated surfaces and compositions, have great application potentials in establishing biological logic systems. However, the reported Janus nanoparticles without large pores are incompatible for the loading of large bio-molecules, limiting their usages as biological logic gates. Herein, an emulsion-oriented assembly approach is demonstrated for the fabrication of highly-uniform Janus double-spherical MSN\&mPDA (MSN = mesoporous silica nanoparticle, mPDA = mesoporous polydopamine) nanoparticles with dual large and tunable mesopores. In this novel approach, oil droplets first interact with MSNs to form double-spherical structures, and then orient the selective-encapsulation of mPDA, leading to the final Janus double-spherical MSN\&mPDA nanoparticles. The delicate Janus nanoparticle possesses a spherical MSN with a uniform size of $\sim 150 \mathrm{~nm}$ in diameter, and a mPDA hemisphere with a diameter of $\sim 120 \mathrm{~nm}$. The dual-mesoporous nanoparticles possess surface area of $\sim 250 \mathrm{~m}^{2} \mathrm{~g}^{-1}$ and also a high pore volume of $\sim 0.56 \mathrm{~cm}^{3} \mathrm{~g}^{-1}$. In addition, the mesopore size in MSN compartment is tunable from $\sim 3$ to $\sim 25 \mathrm{~nm}$, while the mPDA compartments possess mesopores with the diameter ranging from $\sim 5$ to $~$ $50 \mathrm{~nm}$. Due to varied chemical properties and mesopore sizes in the two compartments, selective loading of guests in different compartments of the Janus mesoporous nanoparticles can be achieved. Based on such selective loading, single-particle-level biological logic gates with YES, OR and AND logics have been successfully established for the first time. We believe such work paves the way for constructing advanced asymmetric nanomaterials and realizing complex stimuli-responsive systems.

\section{Introduction}

Mesoporous nanomaterials with large surface area, high pore volume, tunable pore sizes and mesostructures have widely been used in various fields ${ }^{1}$, including catalysis, ${ }^{2,3}$ energy conversion and storage, ${ }^{4-7}$ biomedicine ${ }^{8-12}$ etc. Mesoporous nanoparticles with unique morphologies and structures have been fabricated during the past decades, enabling great enhancements in application properties. ${ }^{13-16}$ Yet still, the single-compartment mesoporous nanoparticles fail to face demands from intricate applications such as biological logic gates, multi-model drug release, etc., as they are unable to provide multiple storage spaces in one single nanoparticle.

Mesoporous Janus nanoparticles, due to the independent mesoporous compartments, spatial isolated surfaces and compositions, have attracted a wide range of attention. ${ }^{14-19}$ To date, a series of asymmetric mesoporous nanoparticles have been fabricated, including Janus, ${ }^{20}$ multipod, ${ }^{21}$ winding, ${ }^{19}$ nanotruck, ${ }^{22}$ di-block \& tri-block, ${ }^{23}$ etc., and various advanced applications have been designed based on the separated mesoporous compartments, such as cascade catalysis, ${ }^{24,25}$ multi-model drug delivery ${ }^{20}$ and so on. Currently, the fabrication of Janus mesoporous nanomaterials is mainly based on the anisotropic assembly of mesoporous compartments, requiring the dual compartments of the Janus nanoparticles to have distinct meso-structures. So, most of the Janus nanoparticles reported previously contain at least one compartment with nanocube or nanorod morphology, such as sphere-cube, sphere-rod, cube-rod 
structured Janus nanocomposites. It is still a great challenge to synthesis the Janus mesoporous nanoparticles with identical meso-structured compartments, impeding the formation of diverse structures such as peanut-like double-spherical architecture. Also, the specific meso-structure further restricts the adjustment of the pore size, so the obtained Janus mesoporous nanoaprticles possess only small mesopores $(<3 \mathrm{~nm})$. The limited pore sizes of the Janus mesoporous nanoparticles hinder their loading capacity of large-sized functional guests such as bio-macromolecules, hampering their application potentials in fields. Therefore, its urgent to develop new strategies for the fabrication of Janus mesoporous nanoparticles with novel architectures and tunable pore sizes.

Biological logic gates utilize the information processing reactions between biomolecules to establish electronic devices like internal logic, therefore greatly expanding the potential applications of biomolecular engineering. The biological logic gates are usually established based on large-sized functional bio-macromolecules, such as nucleic acids, ${ }^{26-29}$ proteins/ enzymes ${ }^{30,31}$ and supramolecular valves $^{32-34}$, and a series of biological logic gates have been built in forms of gel, ${ }^{31}$ polymer, $^{26}$ tablet $^{35}$ and $^{2}$ so on, which are mainly in the form of macroscopic mixtures. Establishing biological logic gates at single-particle-level would be much desired for advanced applications, which is yet hindered by complicated architecting in one single nanoparticle. ${ }^{36-38}$ Janus mesoporous nanoparticles' ability to load multiple cargoes in different compartments, effective separating input, internal processing and output units, makes them much favored for establishing single-particle-level biological logic gates. etc. Yet, single-particle biological logic gates built on small-pore Janus nanoparticles suffers from the low loading capacity of bio-macromolecules and the consequent inefficiency. Thus, developing biological logic systems at a single-particle level calls for Janus mesoporous nanoparticles with novel architectures.

Herein, the mesoporous Janus double-spherical nanoparticles MSN\&mPDAs (MSN = mesoporous silica nanoparticle, $\mathrm{mPDA}=$ mesoporous polydopamine) with tunable dual large mesopores are synthesized for the first time, which is based on a novel emulsion-oriented assembly strategy (Fig. 1a). In this novel approach, oil droplets first interact with MSNs to form double-spherical structures, and then orient the selective-encapsulation of mPDA on the oil droplet, leading to the final Janus doublespherical MSN\&mPDA nanoparticles. The obtained mesoporous Janus MSN\&mPDA nanoparticles possess a double spherical morphology, each nanoparticle is made up of one MSN full nanosphere with a uniform diameter of $\sim 150 \mathrm{~nm}$ and a mPDA hemisphere with $\sim 120 \mathrm{~nm}$ in diameter. The double-spherical Janus mesoporous nanoparticles possess a surface area of $\sim 250 \mathrm{~m}^{2} \mathrm{~g}^{-1}$ and also high pore volume of $\sim 0.56 \mathrm{~cm}^{3} \mathrm{~g}^{-1}$. These nanoparticles possess large and tunable mesopores in both MSN and mPDA compartments with different feature for the hydrophibic and hydrophiphilic. The size of the mesopores in the MSN domain is tunable from 3 to $25 \mathrm{~nm}$, and the mPDA section possesses mesopores with an alterable diameter ranging from $\sim 5$ to $50 \mathrm{~nm}$. Utilizing the varied physicochemical properties (surface charge, conjugated structure, etc.) in the two compartments and the large tunable mesopores of the unique double-spherical nanostructure, various enzymes, supramolecular nanovalves and model molecules are selectively loaded in different compartments of the Janus MSN\&mPDA nanoparticles, and biological logic gates with YES, OR and AND logics are successfully established for 
the first time (Fig. 1b-d). This is the first demonstration of single-nanoparticle-level versatile platform for such biological-logic system establishments. The developed mesoporous Janus MSN\&mPDA nanoparticles with tunable large mesopores and unique asymmetric double-spherical structure provide a new platform for modular bio-computing system construction.

\section{Results}

Spherical mesoporous silica nanoparticles (MSNs) with a uniform diameter of $\sim 150 \mathrm{~nm}$ and a radials mesopore channel of $\sim 8 \mathrm{~nm}$ are synthesized through a bi-phase method by using cetyltrimethylammonium bromide (CTAB) as a structure-directing agent (Supplementary Fig. 1) ${ }^{41}$. After the extraction of CTAB from the MSNs using solvent backflow method (Supplementary Fig. 2), the Janus MSN\&mPDA nanoparticles are formed through an emulsion-oriented assembly of 1,3,5trimethylbenzene (TMB), Pluronic ${ }^{\circledR}$ F-127, CTAB and polydopamine (PDA) on the pre-made MSNs (Fig. 2a). Transmission electron microscopy (TEM) and scanning electron microscopy (SEM) images show that one mPDA hemisphere with a diameter of $\sim 120 \mathrm{~nm}$ and a radial mesopore channel is grown on each MSN, forming the double-spherical Janus MSN\&mPDA nanoparticle (Fig. 2b \& c). Large-scale TEM

(Fig. 2d) and SEM (Supplementary Fig. 3) images demonstrate that the obtained Janus nanoparticles are very uniformly constructed and well dispersed. Nitrogen sorption isotherms of the obtained dualmesoporous MSN\&mPDA Janus nanoparticles show two capillary condensation steps in the relative pressure $P / P_{0}$ ranges of $0.4-0.6$ and $0.8-0.9$, evidencing the co-existence of two sets of uniform mesopores (Supplementary Fig. 4) ${ }^{42,43}$. The Brunauer-Emmett-Teller surface area is measured to be about $243 \mathrm{~m}^{2} \mathrm{~g}^{-1}$ with a high pore volume of $\sim 0.56 \mathrm{~cm}^{3} \mathrm{~g}^{-1}$. The pore size distribution calculated by using Barrett-Joyner-Halenda (BJH) model demonstrates two sets of narrow-distributed mesopores at $\sim 7.8$ and $\sim 14 \mathrm{~nm}$, respectively (Fig. 2f), which match well with the TEM observations (Fig. $\mathbf{2 g}$ \& h). Different elements in the MSN domain (silicon) and mPDA hemisphere (carbon \& nitrogen) can be identified by element mapping (Fig. 2e), further demonstrating the unique asymmetric double-spherical morphology. Such Janus topology as well as the mesoporous structure can be well retained after the calcination at $700{ }^{\circ} \mathrm{C}$ under atmosphere, forming mesoporous Janus MSN\&mC double-spherical nanoparticles (Supplementary Fig. 5). Inorganic functional nanoparticles can also be incorporated into the MSN compartments of the dual-mesoporous Janus structure. For example, UCNP@mSiO ${ }_{2} \& \mathrm{mPDA}$ (UCNP = upconversion nanoparticle, Supplementary Fig. 6) and

$\mathrm{Fe}_{3} \mathrm{O}_{4} @ \mathrm{mSiO}_{2} \& \mathrm{mPDA}$ (Supplementary Fig. 7), providing the Janus structures with various functionalities such as light and magnetism.

The precise manipulation of the pore size in both compartments of the Janus double-spherical nanoparticles can be achieved by varying the type of surfactant and oil phase ${ }^{41,44-46}$. Uniform MSNs with the pore size of $\sim 3,8,15$ and $25 \mathrm{~nm}$ (denoted as $\mathrm{MSN}_{\mathrm{x}}, \mathrm{x}=$ diameter of pore size, nanometer as units) are synthesized by changing the stirring speed and replacing the oil phase from cyclohexane to octadecene and chlorobenzene (Supplementary Fig. 8). The pore size of the mPDA hemi-spherical nanoparticles is also tuned in a range of $5 \sim 50 \mathrm{~nm}$ (denoted as $\mathrm{mPDA}_{\mathrm{y}} \mathrm{y}=$ diameter of pore size, 
nanometer as units) by varying the surfactant from Pluronic ${ }^{\circledR}$ F-127 to P-123 and F108 (Supplementary Fig. 9). So, the mesopore diameters of both MSN and mPDA compartments of the Janus peanut-like nanoparticles can be tuned individually. As shown in Fig. 3, sixteen kinds of the mesoporous $\mathrm{MSN}_{\mathrm{x}} \& \mathrm{mPDA}_{\mathrm{y}}$ Janus double-spherical nanocomposites with controllable pore sizes can be synthesized: the size of mesopores in the MSN domains is tuned to be $\sim 3,8,15$ and $25 \mathrm{~nm}$, while the mesopores of the mPDA domains can be controlled to be $5,15,25,50 \mathrm{~nm}$, respectively. TEM images of the samples with different pore size on both compartments demonstrate the unique Janus nanostructure with tunable dual large mesopores (Fig. 3), which can be assembled to be an extraordinary database. Two groups of nitrogen sorption isotherms are conducted, one group is $\mathrm{MSN}_{8} \& \mathrm{mPDA} \mathrm{A}_{\mathrm{y}}$ Janus nanoparticles with fixed mesopores in the MSN compartment and varied pore sizes in MPDA compartment (Supplementary Fig. 10), and another group is $\mathrm{MSN}_{x} \& \mathrm{mPDA}_{15}$ with varied pore sizes in the MSN compartment (Supplementary Fig. 11). All the nitrogen sorption isotherms are typical type-IV curves with a rapid increase of adsorption volume in double intervals, evidencing the existence and controllability of the dual-mesoporous structure. This is the first demonstration of dual-mesoporous Janus nanoparticles that the diameter of mesopores in both compartments can be tuned precisely and in a large range.

Based on the difference in surface properties between silica and PDA and also the tunable mesopore sizes, selectively loading of multiple guests in various compartments of the Janus mesoporous nanoparticles can be achieved. Typically, Doxorubicin (DOX) is selectively loaded in the mesopore channels of the fully-spherical MSN compartments through a pH-induced selectivity (Fig. 4a). In the neutral environment, DOX molecules are absorbed by both of the MSN and MPDA. DOX molecules are loaded into the MSN compartments due to the capillary effect of small-sized mesopore channels ${ }^{26,47}$, and their attraction towards mPDA is mainly attributed to $\pi-\pi$ stacking ${ }^{48}$. However, at a weak acid condition $(\mathrm{pH}=5)$, both PDA and DOX molecules shift to positive charge ${ }^{48,49}$, the zeta potential of PDA shifts from $-24 \mathrm{mV}$ at neutral $\mathrm{pH}$ to $13 \mathrm{mV}$ at $\mathrm{pH}=5$. This charge repulsion makes the DOX molecules unable to be absorbed by mPDA $\left(<10 \mathrm{mg} \mathrm{g}^{-1}\right)$. On the other hand, the isoelectric point of silica is at the $\mathrm{pH}$ of $\sim 2$, at a pH of 5.5, MSN still possess a negative charge, thus DOX can be loaded into MSNs at a capacity of $\sim 50 \mathrm{mg} \mathrm{g}^{-1}$. Therefore, selective loading of DOX in the MSN compartment can be realized (Supplementary Fig. 12). Enzymes are also selectively loaded in the MPDA domains based on the difference in pore size between the two compartments (Fig. 4b). When using the Janus $\mathrm{MSN}_{8} \& \mathrm{mPDA}_{15}$, the mesopore channels in the MSN compartment with a diameter of $\sim 8 \mathrm{~nm}$ is smaller than the hydrodynamic size of enzymes (e.g. glucose oxidase $=$ Gox have a size of $6.0 \mathrm{~nm} \times 5.2 \mathrm{~nm} \times 7.7$ $\mathrm{nm}^{50}$ ), the loading amount of Gox in the MSN compartments is below the detection limit. The mesopores in the mPDA compartment have sufficient size of $15 \mathrm{~nm}$, and the Gox loading amount is $30 \mathrm{wt} \%$. However, when $\mathrm{MSN}_{25} \& \mathrm{mPDA}_{15}$ nanoparticles with silica mesopore diameter of $15 \mathrm{~nm}$, which is larger than that for the enzymes are used, enzymes are also loaded into the MSN compartment ( $16 \mathrm{wt} \%)$, unable to achieve selective loading. Thus, the different pore size on the two sides of the Janus architecture enables selective loading of enzymes on the mPDA compartment. 
Based on such selective loading of DOX and enzyme in the MSN and mPDA domains, respectively, biological logic gates are established based on the representative $\mathrm{MSN}_{8} \& \mathrm{mPDA}_{15}$ Janus mesoporous nanoparticles (Fig. 5a). Benzimidazole functionalized MSNs with a pore size of $\sim 8 \mathrm{~nm}$ are first fabricated and used for the synthesis of the Janus MSN\&mPDA nanoparticles with a mesopore size of $\sim 15 \mathrm{~nm}$ in PDA compartment. Then $\beta$-cyclodextrin-based supramolecular nanovalves are modified on the MSNs through the benzimidazole- $\beta$-cyclodextrin supramolecular assembly. The benzimidazole- $\beta$-cyclodextrin supramolecular interaction is strong at neutral $\mathrm{pH}$, but breaks in acid condition ${ }^{39,40}$. DOX molecules are selectively loaded in the MSN mesopore channels based on $\mathrm{pH}$-induced selective loading method, and function as the "output signal". The enzymes with different catalytic properties are selectively loaded in the mPDA compartment based on the pore size induced selective loading method, enabling varied logic operations (Supplementary Fig. 13). The enzyme reacts with the substrates ("input signal"), and the products of this biological process are "transmitted" to the supramolecular nanovalves to determine whether or not to open the nanovalves. Thus, the MSN and mPDA are two "processing units" that together form an "internal processor", which sequentially deliver the "input signal" through a series of biochemical reactions to the final "output signal". In such logic gate systems, pH-responsive benzimidazole$\beta$-cyclodextrin-based supramolecular nanovalves on the silica mesopore channels are used as the "lock" to block the release of DOX ${ }^{51,52}$. The products from the reactions between input signal molecules and enzymes selectively loaded in mPDA act as the "key". The nanovalves are "unlocked" only when correct input signal molecules that react with enzymes to form acid are selected as the input signal, opening the "lock" and releasing the loaded DOX as the " 1 " state output signal.

As shown in Fig. 5b, by selecting different functional enzymes in the "internal processor", varied logic process can be achieved. Based on the different loaded enzymes in the MPDA compartment, three different logic gates: YES, OR and AND are established using the large-mesoporous MSN\&mPDA Janus nanoparticles as a general platform (Supplementary Fig. 14). The controlled release of the output signal DOX molecules in these three biological logic gates is carefully analyzed (Supplementary Fig. 15). When being loaded with glucose oxidase (Gox), the input signal (glucose) is catalyzed by Gox to form gluconic acid, lowering the $\mathrm{pH}$, leading to functionalization of the signal converter (opening of the supramolecular nanovalves), and thus the output of signal (release of DOX). Such a response represents the basic YES logic. Logical responsive release of DOX from the dual-mesoporous MSN\&mPDA Janus nanoparticles can be detected upon the addition of glucose (Fig. 5c). Without the presence of glucose, the pHresponsive benzimidazole- $\beta$-cyclodextrin-based supramolecular nanovalves on the silica mesopores are tightly sealed, and the release percentage of DOX is only $\sim 8 \%$, unable to reach the threshold for " 1 " output signal. Other biological logic gates follow the same logical response that only the correct input signals may trigger the internal processing reactions between materials loaded in the two compartments of the dual-mesoporous nanoparticle, resulting in the "1" output signal. When both Gox and esterase are loaded on the mPDA compartment, besides glucose, esters such as ethyl butyrate also triggers the acidforming reaction, leading to " 1 " output. The release percentage of DOX upon ethyl butyrate is $\sim 50 \%$, and that for glucose is $\sim 30 \%$, both successfully functioning as the "1" output signal. This is a typical OR logic (Fig. 5 d). When glucose dehydrogenase (GDH) is used, GDH responds only to the simultaneous 
presence of both glucose and nicotinamide adenine dinucleotide $\left(\mathrm{NAD}^{+}\right)$. The two matters alone cannot trigger an "1" output, the DOX release rates are only $10 \%$ and $~ 15 \%$, unable to reach the threshold value for " 1 " output signal. When both glucose and NAD" are used as the input signals, the DOX release percentage can reach $~ 50 \%$, successfully establishing an AND logic gate (Fig. 5e). These results clearly indicate the successful establishment of various biological logic gates, demonstrating the ability of the dual-mesoporous MSN\&mPDA Janus nanoparticles as a substance for modular bio-computing system constructions.

\section{Discussion}

The mPDA compartment is anisotropically grown on the surface of the MSN spheres in an emulsion system formed by water, ethanol, TMB, F-127 and CTAB. The amount of CTAB is found to play a crucial role in the formation of the Janus double-spherical nanoparticles. The MSNs are fabricated with a large amount of CTAB in the mesopores, and if they are used directly without extraction of CTAB, MPDA fails to perform the anisotropic assembly, and a uniform MSN@mPDA core@shell structure is formed (Supplementary Fig. 16). When the surfactant CTAB is extracted from the MSNs and without further addition of CTAB during the growth of MPDA domain, mPDA refuses to grow on the surface of MSNs leading to phase separation (Supplementary Fig. 17a). When a suitable amount of CTAB (1 mg $\mathrm{mL}^{-1}$ ) is introduced, the Janus structure is achieved (Supplementary Fig. 17b). When further increasing the CTAB amount from 1 to $2.5 \mathrm{mg} \mathrm{mL}^{-1}$, irregular MSN@mPDA structures with multiple mPDA islands are obtained (Supplementary Fig. 17c). Therefore, CTAB is believed to play a crucial role in the anisotropic assembly of uniform mPDA hemispheres to form the unique Janus architecture.

The influence of other factors in the assembly reaction system is also investigated. When decreasing the diameter of the MSNs from 150 to $\sim 100 \mathrm{~nm}$, the Janus structure and the radius of the mPDA hemispheres are maintained still, forming a snowman-like structure. However, when the diameter of MSNs is increased to $\sim 250 \mathrm{~nm}$, multiple mPDA hemispheres are grown on the initial

MSN (Supplementary Fig. 18). When the hydrophilicity of MSNs is decreased through introducing hydrophobic organic functional groups such as ethylene, mPDA nanoparticles have a stronger tendency to phase separate and do not form Janus structure with the MSNs (Supplementary Fig. 19). Shifting the reaction medium (ethanol/water) also changes the assembly behavior of mPDA on

MSN (Supplementary Fig. 20). At a ratio of 1:1, delicate peanut-like Janus nanoparticles are achieved. When increasing water amount to 1:1.5, numerous mPDA hemispheres with a radius of $20 \sim 50 \mathrm{~nm}$ are grown on each MSN nanoparticle, forming a multi-island topology. Further increasing water ratio leads to adsorption of small PDA spheres on MSNs surface. When increasing ethanol amount to a ratio of 1.5:1, several MSN nanoparticles are gathered by one mPDA nanosphere, leading to an agglomeration.

Micrometer-sized agglomerations are found to be formed at the ratio of 2:1. Similar Janus structure can be fabricated using resorcinol formaldehyde resin (RF), forming dual-mesoporous MSN\&mRF Janus structures, indicating the universality of the methodology and that PDA is not the reason for the formation of such architecture (Supplementary Fig. 21). 
The formation process of the dual-mesoporous Janus MSN\&mPDA double-spherical nanoparticles is then investigated (Fig. 6a, b). When the reaction goes on for 2 hours, it can be observed that low-contrast nanosphere is interacted with the solid MSN nanoparticle to form a Janus morphology (Fig. 6c). The nanosphere gradually turns into the mPDA hemisphere as the reaction proceeds (Fig. 6d), finally achieving the dual-mesoporous MSN\&mPDA Janus nanostructure (Fig. 6e).

Based on the above results, we propose the emulsion-orientated assembly process for the formation of the dual-mesoporous MSN\&mPDA Janus double-spherical nanoparticles (Fig. 6f). First, TMB is stabilized by CTAB and F-127 to form an emulsion-like droplet, such TMB nano-droplets interact with the MSNs to form double-spherical Janus structure. In this double-spherical structure, CTAB molecules function as the mediator with its long hydrophobic chain stabilizing TMB droplet, the ammonium side against the hydrophilic MSN. Then the droplets orient the F-127/PDA oligomer micelles to selectively assembly on TMB. The mPDA hemispheres are grown and formed in replace of the TMB droplets, and the peanut-like double-spherical Janus dual mesoporous nanoparticles are obtained.

This emulsion-orientated assembly process is supported by the observed experimental phenomena. CTAB molecules are the "linker" between the TMB droplets and MSNs, therefore minor changes in the amount of CTAB may significantly affect the Janus architecture formation (Supplementary Fig. 16, 17). Appropriate CTAB concentration ( $1 \mathrm{mg} \mathrm{mL}^{-1}$ ) allow the TMB droplets to be adsorbed and "locked" onto the MSNs to form a double-spherical Janus structure, which eventually formed the MSN\&mPDA Janus double-spherical nanoparticles. Without CTAB, TMB droplets cannot be adsorbed onto the MSNs, and mPDA cannot grow on MSNs without the orientation of TMB droplet, resulting in the phase separation. If CTAB is not extracted from the mesopores of MSNs or that the addition of excess CTAB (>2 mg mL $\mathrm{mL}^{-1}$ ) during the assembly reaction, the interaction between the MSN nanoparticle and TMB droplet is stronger than the above mentioned two conditions. The TMB can cover the entire surface of MSN, resulting in the core@shell or multi-island structures (Supplementary Fig. 22, 23). When organic groups are introduced into the silica frameworks, the increased hydrophobicity weakens the interaction of MSNs with surfactants on the TMB droplets (the hydrophobic side of surfactants faces the TMB, the hydrophilic side faces the MSNs), also hindering the formation of particle-droplet double-spherical structure, resulting in phase separation (Supplementary Fig. 19). Water/ethanol ratio changes the size of TMB nano-droplets as well as the particle-emulsion interactions, thus resulting in different architectures (Supplementary Fig. 24).

\section{Conclusions}

Unique mesoporous Janus MSN\&mPDA double-spherical nanoparticles with dual large and tunable mesopores have successfully been synthesized through an emulsion-orientated assembly method. In this novel approach, oil droplets first interact with MSNs to form double-spherical structures, and then orient the selective-encapsulation of mPDA to form Janus double-spherical MSN\&mPDA nanoparticles. The fabricated dual-mesoporous Janus nanoparticles possess a monodispersed MSN nanosphere $(\sim 150 \mathrm{~nm}$ in diameter) with tunable mesopores from $\sim 3$ to $\sim 25 \mathrm{~nm}$, and a mPDA hemisphere with a uniform 
diameter of $\sim 120 \mathrm{~nm}$ and alterable pore size from $\sim 5$ to $\sim 50 \mathrm{~nm}$. This is for the first time realizing the individual and fine manipulation of pore size on both sides of the dual-mesoporous Janus doublespherical nanomaterials. Based on the unique asymmetric double-spherical morphology and dual-largetunable-mesopores, biological logic gates with YES, OR and AND logics are successfully established for the first time, demonstrating the ability of the Janus nanoparticles as the substance for modular biocomputing system construction. Such Janus MSN\&mPDA nanoparticles with dual large and tunable mesopores and selective functionalization are highly extendable and have great potential of being a multifunctional platform not only for biological logic gates but also for catalysis, energy storage, sensing, and environmental remediation.

\section{Methods}

\section{Fabrication of mesoporous silica nanoparticles (MSN):}

MSNs were synthesized through a bi-phase method according to previous reports with brief modifications. ${ }^{41}$ In a typical synthesis of MSNs with a pore size of $\sim 8 \mathrm{~nm}, 3.00 \mathrm{~g}$ of CTAB, $100 \mu \mathrm{L}$ of triethanolamine, $60.0 \mathrm{~mL}$ of $\mathrm{H}_{2} \mathrm{O}$ were added in a $100-\mathrm{mL}$ volume three-necked flask. The mixture was stirred until clear, then transferred into an oil bath at $60^{\circ} \mathrm{C}$. A Teflon rotor with a length of $2.0 \mathrm{~cm}$ was used and the stirring rate was set to be $150 \mathrm{rpm}$. Then a mixture of $4.0 \mathrm{~mL}$ of TEOS and $16.0 \mathrm{~mL}$ of cyclohexane were added carefully on top of the solution to form a biphase. The reaction was allowed to proceed for $48 \mathrm{~h}$, then centrifuged, washed with water and ethanol. The synthesis of MSNs with other pore sizes are recorded in the Supplementary Information.

\section{Template CTAB extraction from the MSNs:}

The MSNs were dispersed in a $5.0 \mathrm{mg} \mathrm{mL}^{-1} \mathrm{NH}_{4} \mathrm{NO}_{3}$ ethanol solution and refluxed at $60{ }^{\circ} \mathrm{C}$ for $4 \mathrm{~h}$. This process was repeated three times to guarantee the complete extraction of CTAB.

\section{Fabrication of Janus nanoparticles MSN\&mPDA:}

The MSN\&mPDA Janus nanoparticles were fabricated through an emulsion-orientated assembly process. In a typical process, $5.0 \mathrm{mg}$ of MSNs obtained above with CTAB extracted were dispersed in $5.0 \mathrm{~mL}$ of $\mathrm{H}_{2} \mathrm{O}, 5.0 \mathrm{~mL}$ of ethanol. Then, $10.0 \mathrm{mg}$ of CTAB, $200 \mathrm{mg}$ of F-127, $100 \mathrm{mg}$ of dopamine were also added and stirred to form a clear solution. Then $1.0 \mathrm{~mL}$ of TMB was added, the mixture was stirred for $30 \mathrm{~min}$ to form a milky white emulsion, and then $10.0 \mathrm{mg}$ of tris(hydroxymethyl)aminomethane was added. The reaction was left to proceed overnight, then centrifuged and washed with water and ethanol. The synthesis of MSN\&mPDA nanoparticles with other pore sizes are recorded in the Supplementary Information.

\section{Data availability}


Data supporting the findings of this study are available within the article and the associated Supplementary Information Section.

\section{Declarations}

\section{Acknowledgements}

The work was supported by the National Key R\&D Program of China (2018YFA0209401), National Natural Science Foundation of China $(22075049,21875043,22088101,21701027,21733003,21905052$, 51961145403), Key Basic Research Program of Science and Technology Commission of Shanghai Municipality (17JC1400100), Natural Science Foundation of Shanghai (18ZR1404600, 20490710600), Shanghai Rising-Star Program (20QA1401200), NPRP grant NPRP12S-0309-190268 from the Qatar National Research Fund.

\section{Author contributions}

D.Z., X.L. and T.Z. contributed to the conception and design of the experiments, analysis of the data and writing the manuscript. L.C., M.L, R.L, W.C., C.H., S.W., L.D., A.E., F.Z. and X.L. assisted T.Z. for the synthesis of materials and the data collection and analysis. All authors contributed to the discussion and manuscript preparation.

\section{Additional information}

Supplementary Information accompanies this paper at http://www.nature.com/

\section{Competing interests}

The authors declare no competing interests.

\section{References}

1 Ryoo, R. Birth of a class of nanomaterial. Nature. 575, 40-41 (2019).

2 Joo, S., Park, J., Tsung, C., Yamada, Y., Yang, P. \& Somorjai, G. Thermally stable Pt/mesoporous silica core-shell nanocatalysts for high-temperature reactions. Nat. Mater. 8, 126-131 (2009).

3 Parlett, C. M. et al. Spatially orthogonal chemical functionalization of a hierarchical pore network for catalytic cascade reactions. Nat. Mater. 15, 178-182 (2016).

4 Li, W., Liu, J. \& Zhao, D. Y. Mesoporous materials for energy conversion and storage devices. Nat. Rev. Mater. 1, 16023 (2016). 
5 Lan, K. et al. Precisely designed mesoscopic titania for high-volumetric-density pseudocapacitance. J. Am. Chem. Soc. doi.org/10.1021/jacs.1c03433 (2021)

$6 \mathrm{Zu}$, L. et al. Mesoporous materials for electrochemical energy storage and conversion. Adv. Energy Mater. 10, 2002152 (2021).

7 Wang, C. et al. Molecular design strategy for ordered mesoporous stoichiometric metal oxide. Angew. Chem. Int. Ed. 58, 15863-15868 (2019).

8 Li, Z., Barnes, J., Bosoy, A., Stoddart, J. \& Zink, J. Mesoporous silica nanoparticles in biomedical applications. Chem. Soc. Rev. 41, 2590-2605 (2012)

9 Chen, W., Glackin, C. A., Horwitz, M. A. \& Zink, J. Nanomachines and other caps on mesoporous silica nanoparticles for drug delivery. Acc. Chem. Res. 52, 1531-1542 (2019).

10 Chen, Y. \& Shi, J. Chemistry of mesoporous organosilica in nanotechnology: molecularly organicinorganic hybridization into frameworks. Adv. Mater. 28, 3235-3272 (2016).

11 Liu, J., Liu, T., Pan, J., Liu, S. \& Lu, G. Advances in multicompartment mesoporous silica micro/nanoparticles for theranostic applications. Annu. Rev. Chem. Biomol. 9, 389-411 (2018).

12 Nguyen, T. L., Choi, Y. \& Kim, J. Mesoporous silica as a versatile platform for cancer immunotherapy. Adv. Mater. 31, e1803953 (2019).

13 Qiu, P., Ma, B., Hung, C. T., Li, W. \& Zhao, D. Spherical mesoporous materials from single to multilevel architectures. Acc. Chem. Res. 52, 2928-2938 (2019).

14 Zhao, T. C., Elzatahry, A., Li, X. M. \& Zhao, D. Y. Single-micelle-directed synthesis of mesoporous materials. Nat. Rev. Mater. 4, 775-791 (2019).

15 Li, W., Yue, Q., Deng, Y. \& Zhao, D. Ordered mesoporous materials based on interfacial assembly and engineering. Adv. Mater. 25, 5129-5152 (2013).

16 Zhao, T. C. et al. Interfacial assembly directed unique mesoporous architectures: from symmetric to asymmetric. Acc. Mater. Res. 1, 100-114 (2020).

17 Chen, C., Xie, L. \& Wang, Y. Recent advances in the synthesis and applications of anisotropic carbon and silica-based nanoparticles. Nano Res. 12, 1267-1278 (2019).

$18 \mathrm{Wu}, \mathrm{Z}$. et al. Janus nanoarchitectures: from structural design to catalytic applications. Nano Today 22, 62-82 (2018).

19 Zhao, T. C. et al. Surface confined winding assembly of mesoporous nanorods. J. Am. Chem. Soc. $142,20359-20367(2020)$ 
$20 \mathrm{Li}, \mathrm{X}$. et al. Anisotropic growth-induced synthesis of dual-compartment Janus mesoporous silica nanoparticles for bimodal triggered drugs delivery. J. Am. Chem. Soc. 136, 15086-15092 (2014).

21 Zhao, T. et al. Surface-kinetics mediated mesoporous multipods for enhanced bacterial adhesion and inhibition. Nat. Commun. 10, 4387 (2019).

22 Wang W. et al. Engine-trailer-structured nanotrucks for efficient nano-bio interactions and bioimagingguided drug delivery. Chem 6, 1-16 (2020).

$23 \mathrm{Li}, \mathrm{X}$. et al. Degradation-restructuring induced anisotropic epitaxial growth for fabrication of asymmetric diblock and triblock mesoporous nanocomposites. Adv. Mater. 29, 1701652 (2017).

24 Yang, T. et al. Dumbbell-shaped bi-component mesoporous Janus solid nanoparticles for biphasic interface catalysis. Angew. Chem. Int. Ed. 56, 8459-8463 (2017).

25 Zhao, T. et al. Spatial isolation of carbon and silica in a single Janus mesoporous nanoparticle with tunable amphiphilicity. J. Am. Chem. Soc. 140, 10009-10015 (2018).

26 Zhang, P. H. et al. A programmable polymer library that enables the construction of stimuli-responsive nanocarriers containing logic gates. Nat. Chem. 12, 381-390 (2020).

27 Lucks, J. B., Qi, L., Mutalik, V. K., Wang, D. \& Arkin, A. P. Versatile RNA-sensing transcriptional regulators for engineering genetic networks. Proc. Natl. Acad. Sci. 108, 8617-8622 (2011).

28 Seelig, G., Soloveichik, D., Zhang, D. Y. \& Winfree, E. Enzyme-free nucleic acid logic circuits. Science 314, 1585-1588 (2006).

29 Win, M. N. \& Smolke, C. D. Higher-order cellular information processing with synthetic RNA devices. Science 322, 456-460 (2008).

30 Strack, G., Ornatska, M., Pita, M. \& Katz, E. Biocomputing security system: concatenated enzymebased logic gates operating as a biomolecular keypad lock. J. Am. Chem. Soc. 130, 4234-4235 (2008).

31 Ikeda, M. et al. Installing logic-gate responses to a variety of biological substances in supramolecular hydrogel-enzyme hybrids. Nat. Chem. 6, 511-518 (2014).

32 Wen, J. et al. Diverse gatekeepers for mesoporous silica nanoparticle based drug delivery systems. Chem. Soc. Rev. 46, 6024-6045 (2017).

33 Park, J. S. et al. Disparate downstream reactions mediated by an ionically controlled supramolecular tristate switch. J. Am. Chem. Soc. 140, 7598-7604 (2018).

34 Karimi, M. et al. Smart nanostructures for cargo delivery: uncaging and activating by light. J. Am. Chem. Soc. 139, 4584-4610 (2017). 
35 Seo J. et al. Nano-bio-computing lipid nanotablet. Sci. Adv. 5, eaau2124 (2019).

36 Nenenson Y. Biomolecular computing systems: principles, progress and potential. Nat. Rev. Genetics 13, 455-468 (2012).

37 Lius B. et al. Engineering chemical communication between micro/nanosystems. Chem. Soc. Rev. 16, 8829-8856 (2021).

38 Chen, C. et al. Bioinspired chemical communication between synthetic nanomotors. Angew. Chem. Int. Ed. 57, 241-245 (2018).

39 Llopis-Lorente, A. et al. Interactive models of communication at the nanoscale using nanoparticles that talk to one another. Nat. Commun. 8, 15511 (2017).

40 Diez, P. et al. Toward the design of smart delivery systems controlled by integrated enzyme-based biocomputing ensembles. J. Am. Chem. Soc. 136, 9116-9123 (2014).

41 Shen, D. et al. Biphase stratification approach to three-dimensional dendritic biodegradable mesoporous silica nanospheres. Nano Lett. 14, 923-932 (2014).

$42 \mathrm{Niu}, \mathrm{D} ., \mathrm{Ma}, \mathrm{Z} ., \mathrm{Li}, \mathrm{Y}$. \& Shi, J. Synthesis of core-shell structured dual-mesoporous silica spheres with tunable pore size and controllable shell thickness. J. Am. Chem. Soc. 132, 15144-15147 (2010).

43 Wei, J., Yue, Q., Sun, Z., Deng, Y. \& Zhao, D. Synthesis of dual-mesoporous silica using non-ionic diblock copolymer and cationic surfactant as co-templates. Angew. Chem. Int. Ed. 51, 6149-6153 (2012).

44 Guan, B. Y., Zhang, S. L. \& Lou, X. Realization of walnut-shaped particles with macro-/mesoporous open channels through pore architecture manipulation and their use in electrocatalytic oxygen reduction. Angew. Chem. Int. Ed. 57, 6176-6180 (2018).

45 Chen, G. et al. General formation of macro-/mesoporous nanoshells from interfacial assembly of irregular mesostructured nanounits. Angew. Chem. Int. Ed. 59, 19663-19668 (2020).

46 Peng, L. et al. Versatile nanoemulsion assembly approach to synthesize functional mesoporous carbon nanospheres with tunable pore sizes and architectures. J. Am. Chem. Soc. 141, 7073-7080 (2019).

47 Vallet-Regi, M., Rámila, A., del Real, R. P. \& Pérez-Pariente, J. A new property of MCM-41: drug delivery system. Chem. Mater. 13, 308-311 (2001).

48 Liu, Q., Yu, B., Ye, W. \& Zhou, F. Highly selective uptake and release of charged molecules by pHresponsive polydopamine microcapsules. Macromol. Biosci. 11, 1227-1234 (2011).

49 Yu, B., Liu, J., Liu, S. \& Zhou, F. Pdop layer exhibiting zwitterionicity: a simple electrochemical interface for governing ion permeability. Chem. Commun. 46, 5900-5902 (2010). 
50 Huo, M., Wang, L., Chen, Y. \& Shi, J. Tumor-selective catalytic nanomedicine by nanocatalyst delivery. Nat. Commun. 8, 357 (2017).

51 Du, L., Liao, S., Khatib, H. A., Stoddart, J. F. \& Zink, J. I. Controlled-access hollow mechanized silica nanocontainers. J. Am. Chem. Soc. 131, 15136-15142 (2009).

52 Coll, C., Bernardos, A., Martinez-Manez, R. \& Sancenon, F. Gated silica mesoporous supports for controlled release and signaling applications. Acc. Chem. Res. 46, 339-349 (2013).

\section{Figures}

a
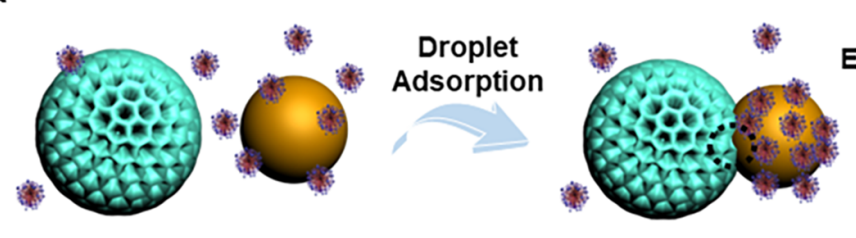

b

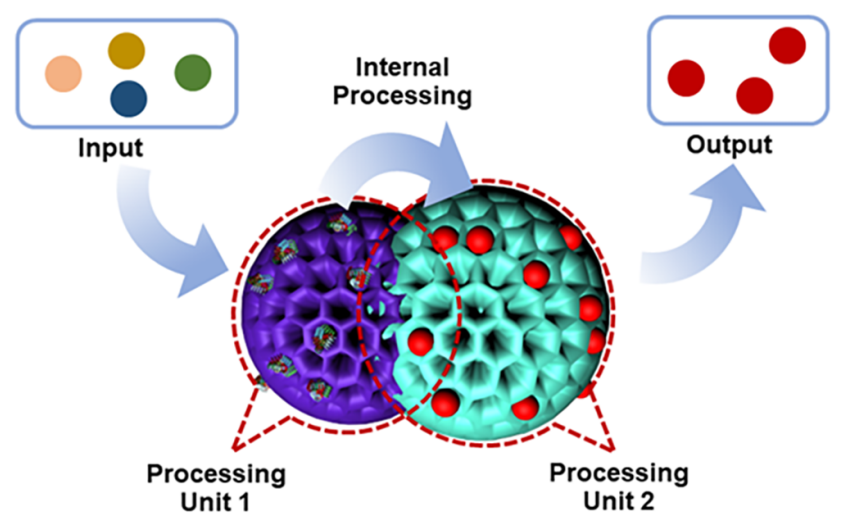

Emulsion Oriented Assembly

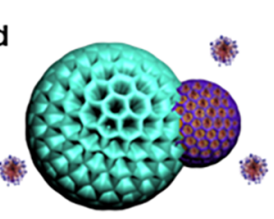

Selective Encapsulation

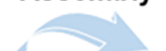

C

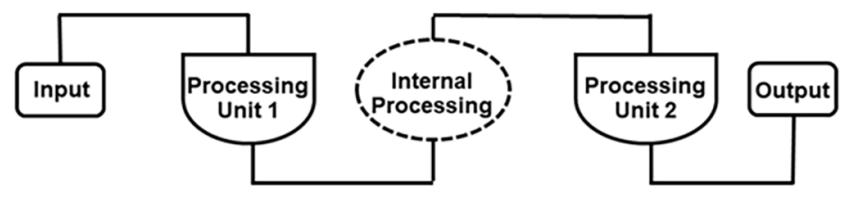

d

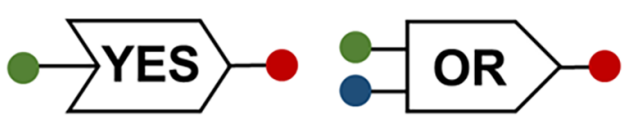

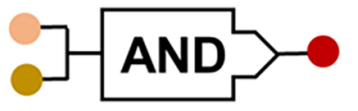

Figure 1

Schematic illustration of the synthesis and biological-logic gates of Janus mesoporous nanoparticles MSN\&mPDA. a) Schematic illustration of the emulsion-orientated assembly process for the synthesis of the Janus double-spherical mesoporous MSN\&mPDA nanoparticles (MSN = mesoporous silica nanoparticle, $\mathrm{mPDA}=$ mesoporous polydopamine). $\mathrm{b}, \mathrm{c}$ ) Representation of the single-particle-level biological logic gate and keypad lock system as a network of the logic gates. d) Representation of the three kinds of logic system established based on the Janus double-spherical mesoporous MSN\&mPDA nanoparticles. 
a
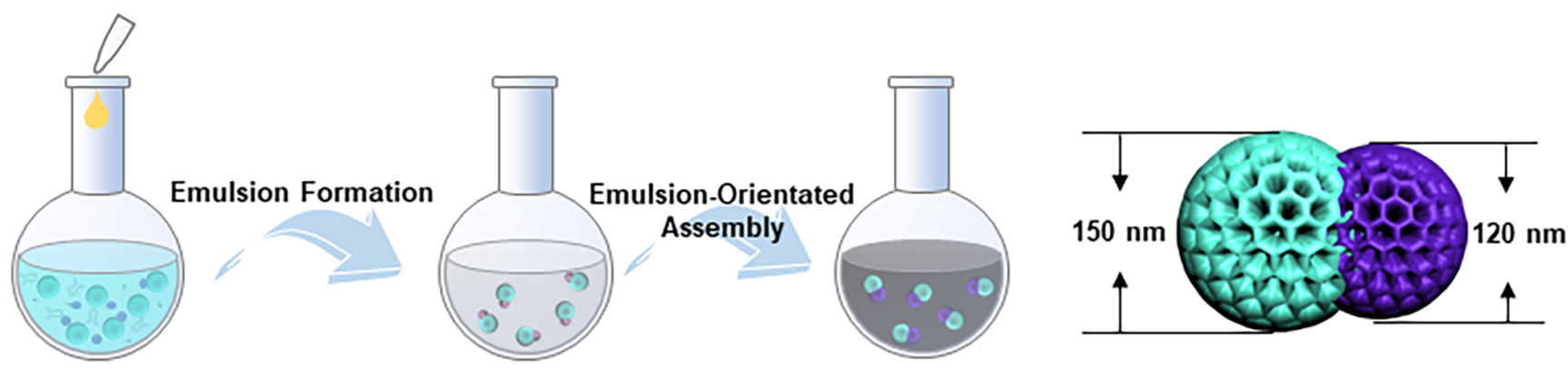

MSN PDA oligomer $\Omega$ F127 $ई$ CTAB TMB

b

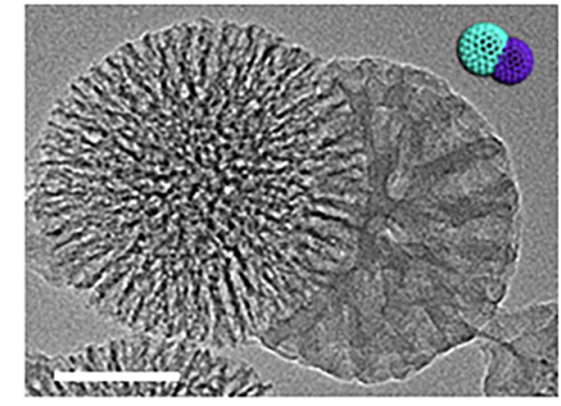

d

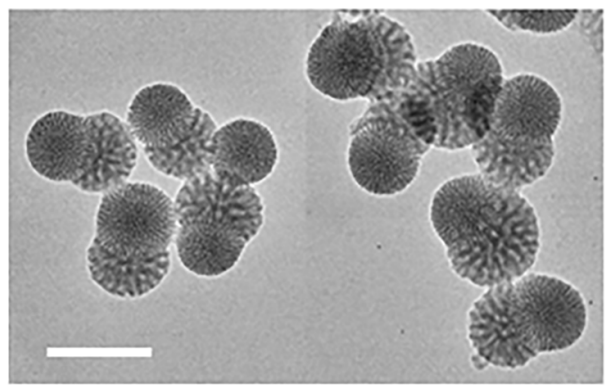

C

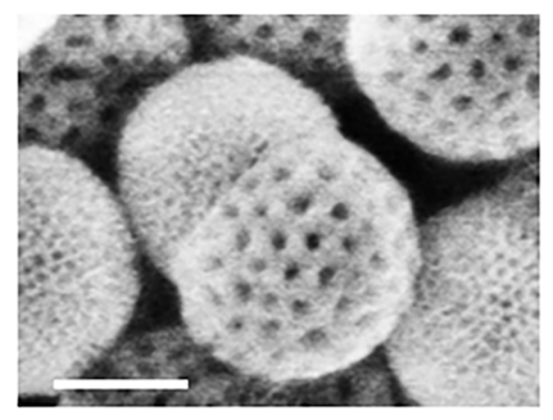

e

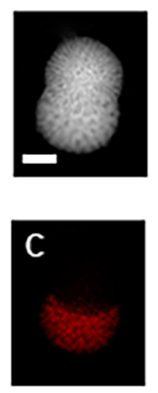

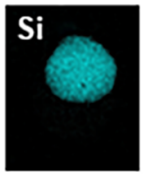
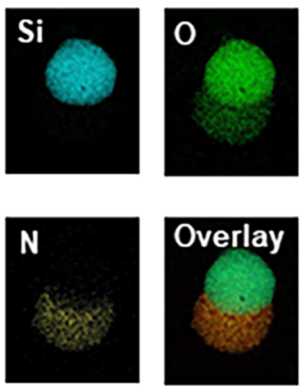

Overlay

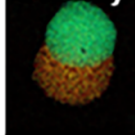

Water\&ethanol

\& Emulsion

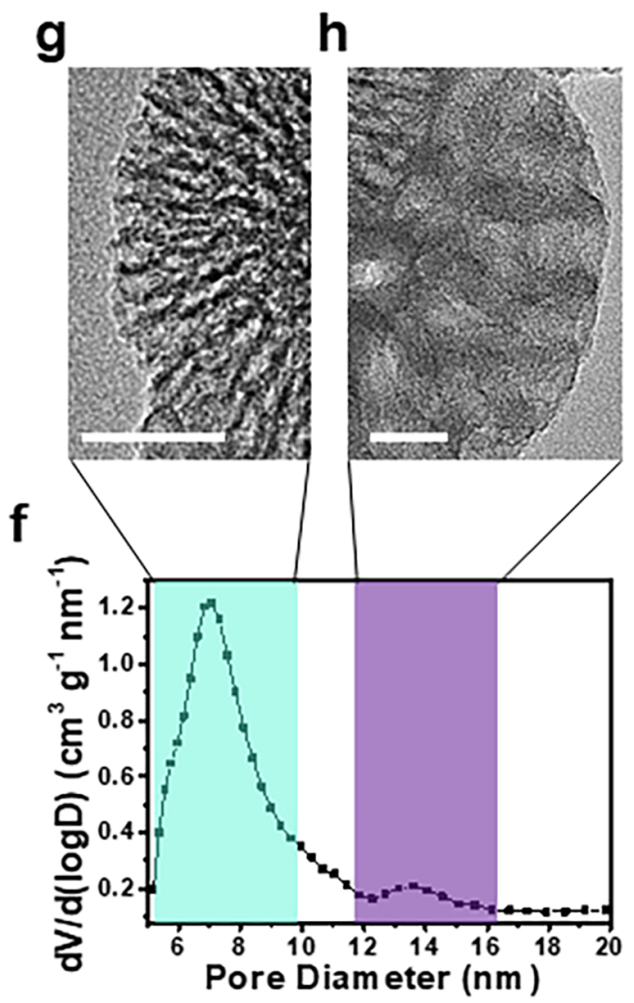

Figure 2

Emulsion-orientated assembly of Janus mesoporous nanoparticles MSN\&mPDA. a) Schematic illustration of the emulsion-orientated assembly process for Janus double-spherical mesoporous MSN\&mPDA nanoparticles with tunable and large mesopores. b, d) transmission electron microscopy (TEM) images with different magnifications, c) scanning electron microscopy (SEM) image, e) dark-field TEM image and element mappings of the dual-mesoporous Janus MSN\&mPDA nanoparticles. f) The pore size distribution of the obtained Janus MSN\&mPDA nanoparticles and high-resolution TEM (HRTEM) images of the mesopores in g) MSN and h) mPDA compartments. Scale bar: $50 \mathrm{~nm}$ for b, 100 $\mathrm{nm}$ for $\mathrm{c}, 200 \mathrm{~nm}$ for $\mathrm{d}, 20 \mathrm{~nm}$ for e, $\mathrm{g}$ and $\mathrm{h}$. 


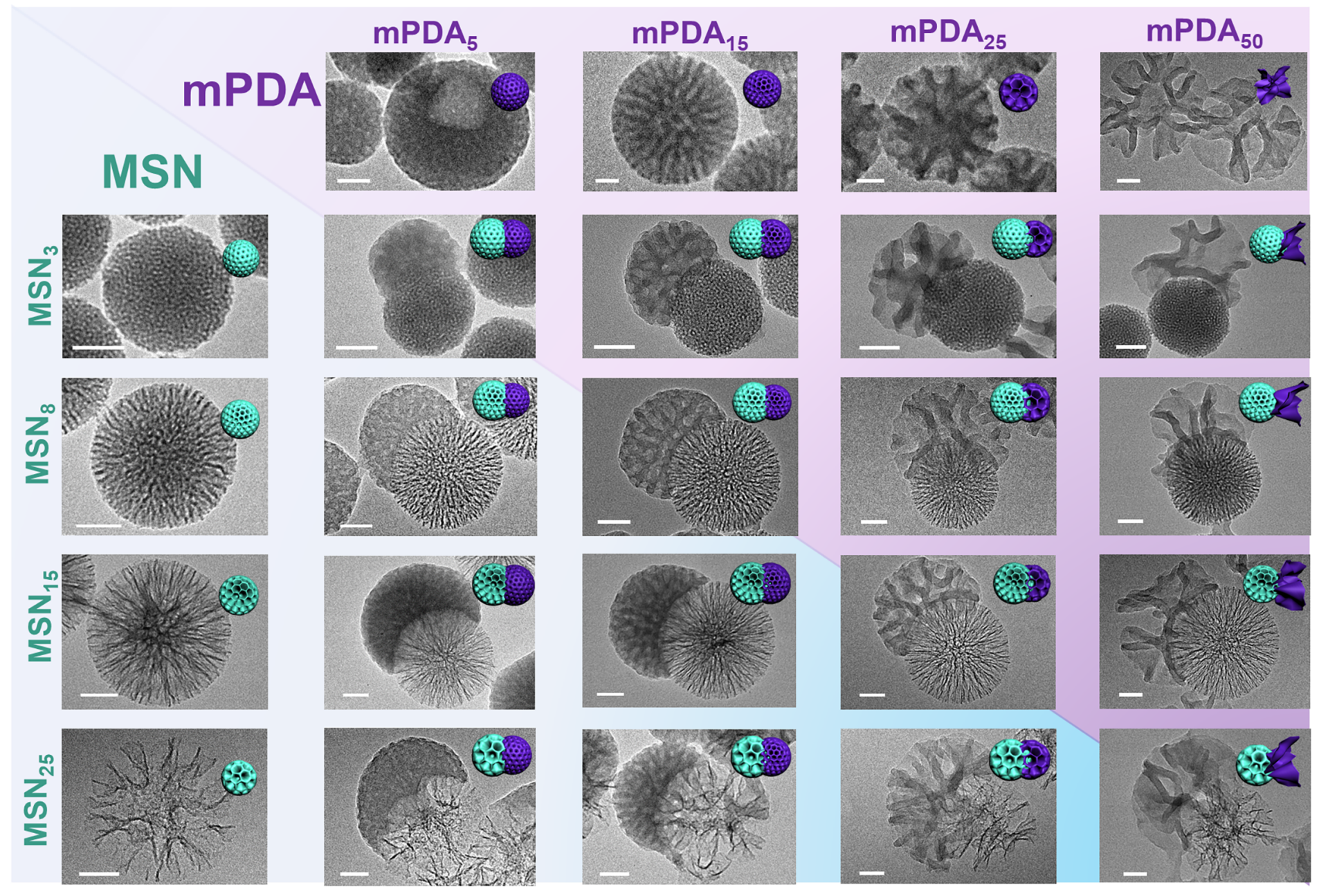

Figure 3

Janus double-spherical MSN\&mPDA nanoparticles with tunable large mesopores. TEM images of the mesoporous silica nanoparticles with different pore sizes (denoted as MSNx, $x=$ pore diameter in $\mathrm{nm}$ ), the mesoporous polydopamine nanoparticles with different pore sizes (denoted as mPDAy, $y=$ pore diameter in $\mathrm{nm}$ ) and dual-mesoporous Janus MSNx\&mPDAy nanoparticles with varied mesopore diameter on both sides of the Janus nanoparticles. Scale bar: $50 \mathrm{~nm}$. 


\section{П-П Interaction}

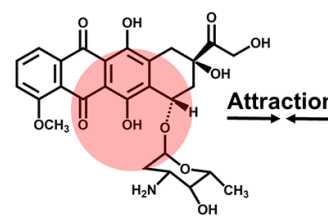

DOX

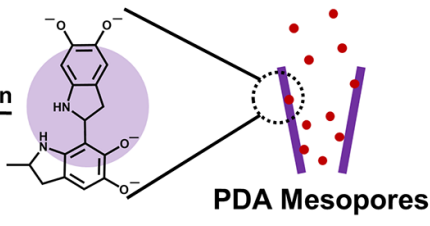

PDA

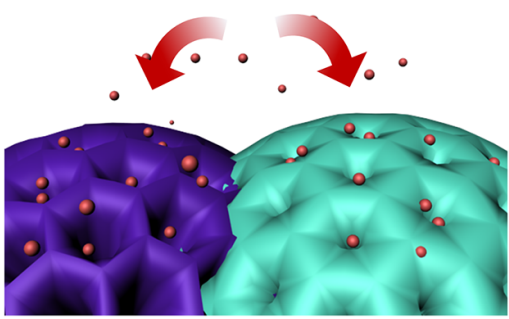

Dual Loading at $\mathrm{pH}=7$

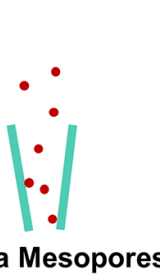

Silica Mesopores

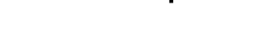

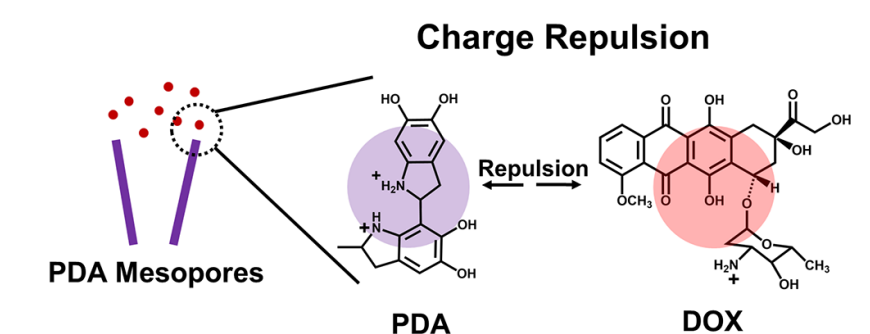

PDA

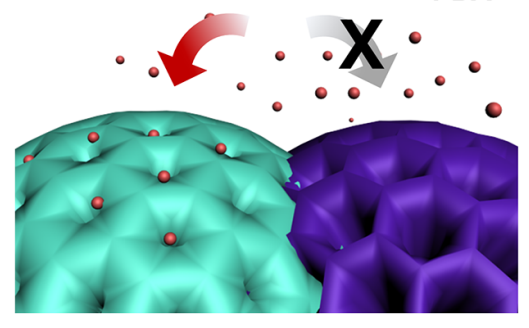

Selective Loading at $\mathrm{pH}=5$

b

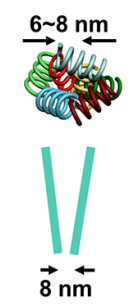

No Loading in Silica Mesopores

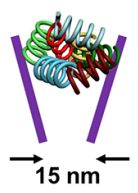

Loading in PDA Mesopores

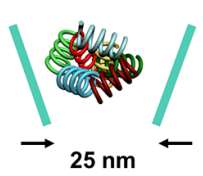

Loading in Silica Mesopores

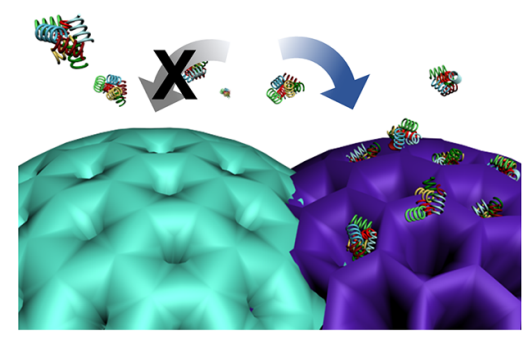

$\mathrm{MSN}_{8}$ \&mPDA 15 Selective Loading

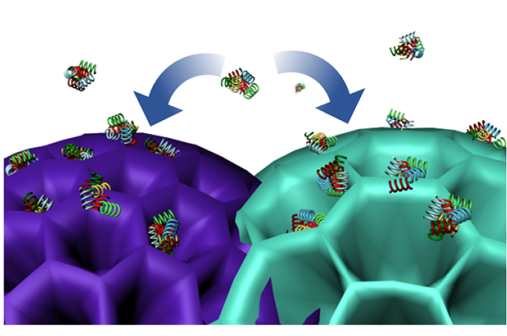

$\mathrm{MSN}_{15} \& \mathrm{mPDA}_{15}$ Non-selective Loading

Figure 4

Schematic illustration of the selective loading based on the size and surface property of the mesopores.

a) Schematic illustration of the $\mathrm{pH}$-induced selective loading of Doxorubicin (DOX) in the MSN compartment based on the different pore environments between MSN and MPDA. DOX molecules are loaded in both MSN and mPDA compartments at neutral $\mathrm{pH}$, but can only be loaded in the MSN mesopores at $\mathrm{pH}=5$, which is due to the charge repulsion between DOX molecules and PDA. b) Schematic illustration of the selective modification of glucose oxidase in the mPDA compartment based on the different pore sizes between MSN and mPDA. As the mesopore size on both sides of the dualmesoporous Janus nanoparticles can be precisely tuned, large-sized enzymes can only be loaded in the mPDA spheres with the mesopores of $15 \mathrm{~nm}$ but not the MSN mesopores with a diameter of $8 \mathrm{~nm}$, therefore achieving selective modification. 
a

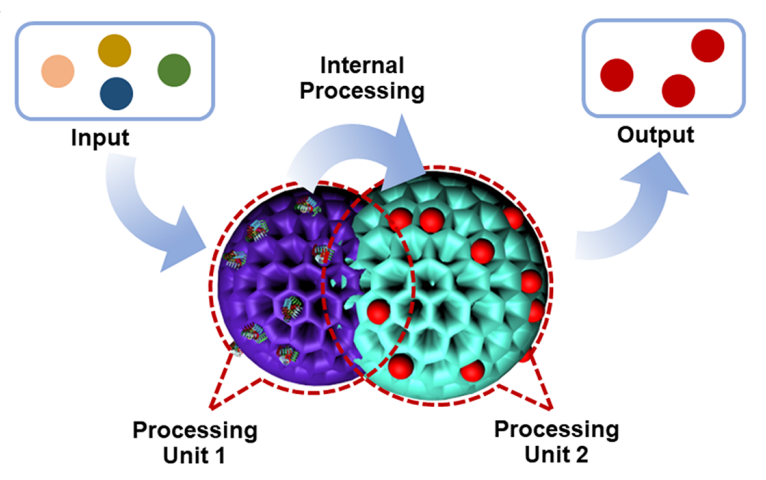

C

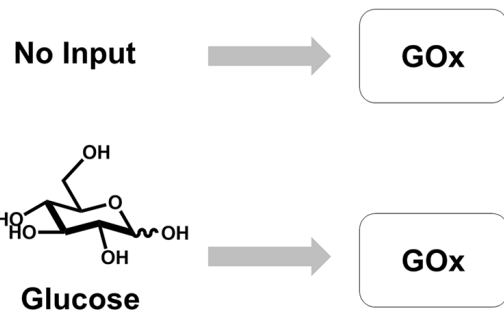

d<smiles>CCCC(=O)CCO</smiles>

Ethyl butyrate

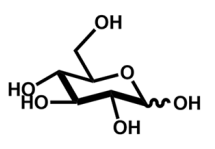

Glucose

e<smiles>CC(=O)C1=CN(C)C=CC1(C)C</smiles>

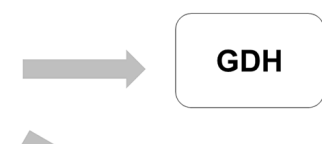

NADH

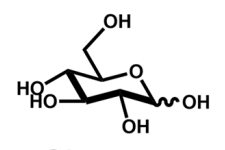

Glucose

GOx \&
Esterase

GOx \&
Esterase

Gluconic Acid

Gluconic Acid

No pH Change

Butyric Acid

No pH Change

Gluconic Acid

GDH

GDH
No pH Change
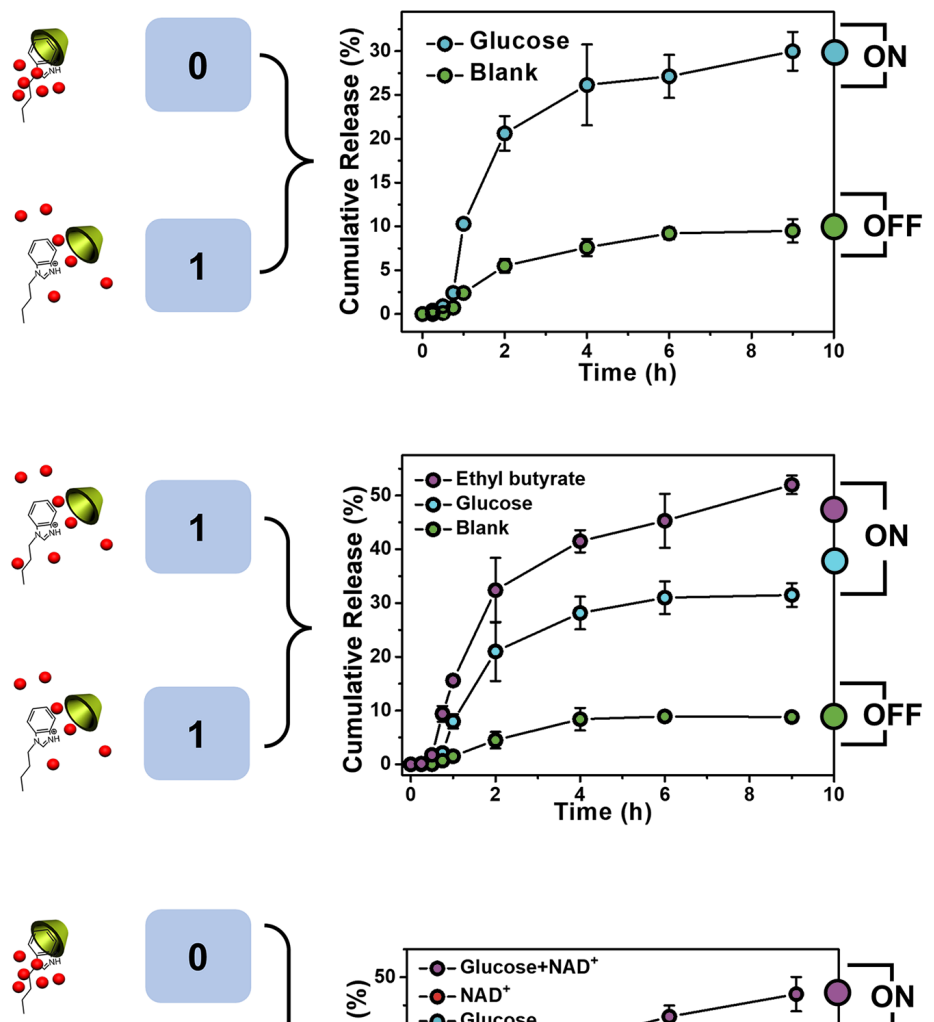

-

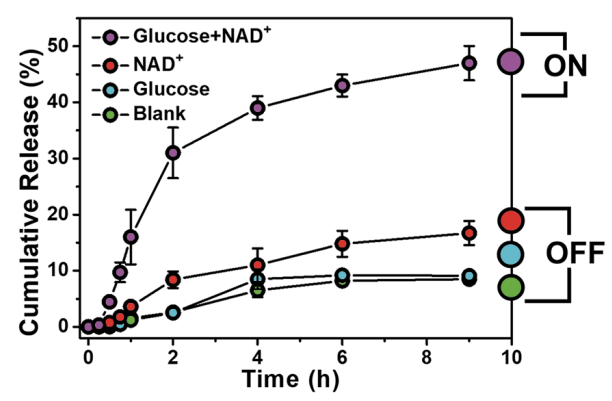

\section{Figure 5}

Janus mesoporous double-spherical nanoparticles based biological logic gates. a) Simplified illustration of Janus mesoporous nanoparticle based biological logic gates. b) Schematic illustration of the three established biological logic gates. Schematic representation of internal stimuli-responsive reactions and output DOX release profile of the reactions in the c) YES, d) OR and e) AND gate. For the YES gate, the output signal from Doxorubicin (DOX) molecules is released when the input signal of glucose molecules 
is catalyzed by glucose oxidase (Gox) into glucose acid, opening the $\mathrm{pH}$-responsive benzimidazole- $\beta$ cyclodextrin nanovalve and triggering the release of the loaded DOX molecules. For the OR gate, Gox and esterase are loaded on the mPDA compartment, both glucose and ethyl butyrate can trigger the formation of acid and thus the opening of the nanovalves, realizing the DOX release. For the AND gate, glucose dehydrogenase (GDH) is modified on the mPDA compartment. Gluconic acid can be formed only in the presence of both glucose and nicotinamide adenine dinucleotide, thus the release of DOX molecules can be achieved only when two inputs are provided together. Error bars with an absolute value smaller than 1 are omitted in the release profile figures for better visualization. The error bars represent mean \pm s.d. derived from $n=3$ groups of tests.

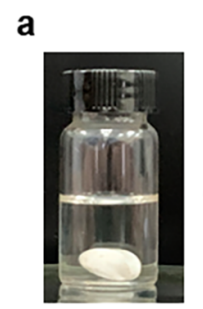

Initial Solution b

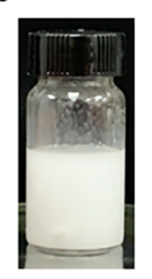

Emulsion c

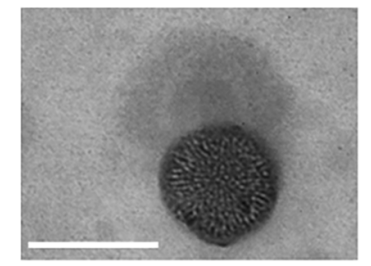

2 h: Particle-oil Heterodimer d

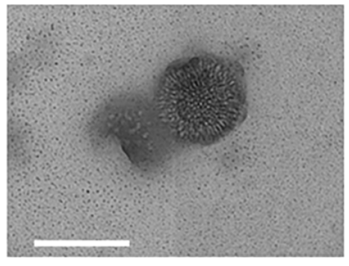

$4 \mathrm{~h}$ : Oriented Assembly e

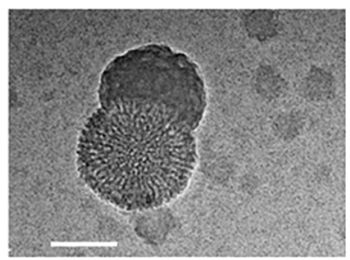

$8 \mathrm{~h}$ : Janus Structure

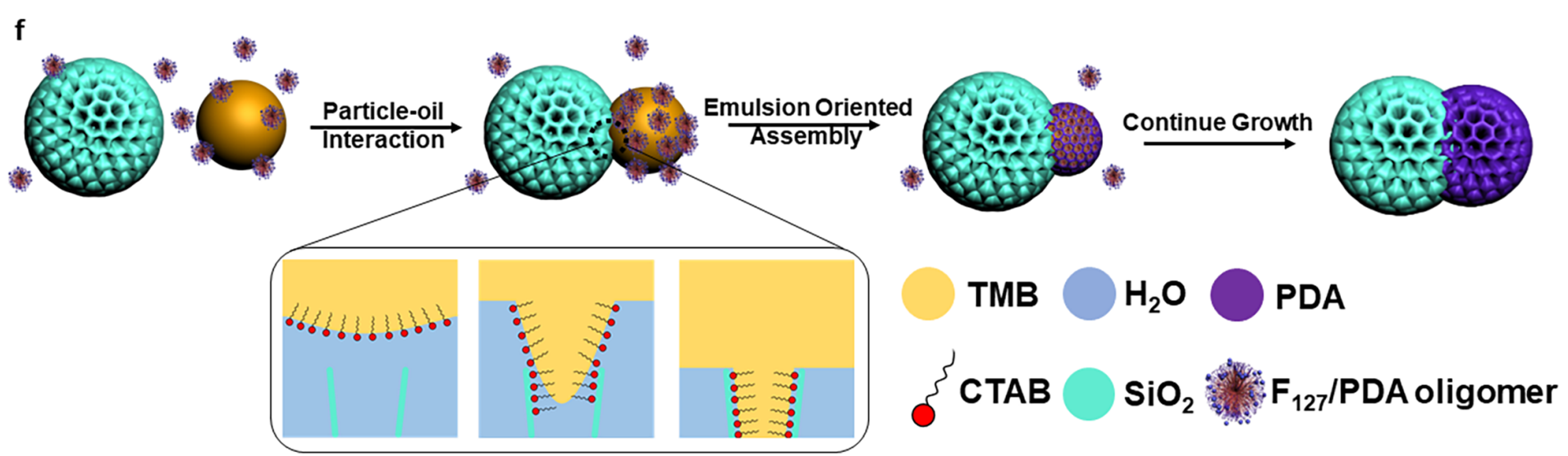

Figure 6

Mechanism of the emulsion-orientated assembly process. Digital photos of the reaction solution a) before and $b$ ) after the addition of TMB. TEM images of the products obtained by directly dropping the reaction mixture on the copper mesh at c) $2 \mathrm{~h}, \mathrm{~d}) 4 \mathrm{~h}$ and e) $8 \mathrm{~h}$. Schematic illustration of the emulsiondirected oriental assembly approach for the fabrication of the dual-mesoporous Janus MSN\&mPDA double-spherical nanoparticles. Scale bar: $200 \mathrm{~nm}$ for c and d, $100 \mathrm{~nm}$ for e.

\section{Supplementary Files}

This is a list of supplementary files associated with this preprint. Click to download.

- Sl.docx 